André Hagenbruch und Miriam Heise

\title{
„suchen“ - ein Prototyp zum Vergleich von Discovery-Diensten
}

Zusammenfassung: Wir beschreiben den Prototypen einer Webanwendung, die zwei unterschiedliche Discovery-Dienste und einen OPAC-basierten Suchmaschinenindex integriert. Zunächst skizzieren wir die technische Architektur, geben dann einen Überblick über die Metadaten und APIs der eingesetzten DiscoveryDienste und berichten im Anschluss von den Nutzererfahrungen, die wir aus dem Feedback der Testpersonen gewonnen haben. Zuletzt geben wir einen Überblick über die Ergebnisse unseres Tests und umreißen die Richtung, in die wir unsere Entwicklung fortführen wollen.

Schlüsselwörter: Discovery-Dienst, Webanwendung, User Experience, Universitätsbibliothek, Ruhr-Universität Bochum

\section{"search" - a prototype to compare resource discovery services}

Abstract: We describe a prototype web application that integrates two different resource discovery systems and an OPAC based search index. Starting from an outline of the technical architecture we give an overview of the metadata and APIs encountered in the discovery systems and then report on the user experience we gleaned from the feedback we got during the testing phase. Finally, we present the conclusions we have drawn from our tests and indicate the directions in which we plan to continue our development.

Keywords: resource discovery service, web application, user experience, university library, Ruhr-University Bochum

DOI 10.1515/bd-2014-0099

André Hagenbruch: andre.hagenbruch@rub.de

Miriam Heise: miriam.heise@rub.de 


\section{Einleitung}

Im Mai 2008 startete die Universitätsbibliothek Bochum das Projekt „Integriertes Bibliotheksportal“", in dem eine Anwendung entstehen sollte, um den Benutzern möglichst viele Datenquellen unter einer einheitlichen Oberfläche zur Recherche auf Basis moderner Suchmaschinentechnologie anbieten zu können. Darüber hinaus sollte die Anwendung auch den aktuellen Verfügbarkeitsstatus des jeweiligen Titels anzeigen und Benutzerfunktionalitäten wie z. B. Vormerkungen oder Kontoverwaltung ermöglichen. Nachdem wir zunächst intensiv OCLC TouchPoint evaluiert hatten, zeichnete es sich ab, dass wir damit nicht alle unsere Ziele erreichen würden. Parallel zu diesem Projekt haben wir eine auf Open-Source-Software basierende Such-Plattform für die Hochschulbibliographie der Ruhr-Universität Bochum (RUB) entwickelt, die so konzipiert war, dass mit geringen Anpassungen unterschiedliche Datenquellen integriert werden konnten. Im September 2011 testeten wir mit ProQuest zum ersten Mal die Möglichkeit, einen Discovery-Dienst in diese Plattform zu integrieren. Da ein Demonstrator recht schnell implementiert werden konnte, entschlossen wir uns, die Produkte Summon von ProQuest und EDS von EBSCO durch Integration über die angebotenen APIs sowie einen Suchmaschinenindex mit unseren Katalogdaten nebeneinander zu evaluieren.

Aufbauend auf den in Jansen et al. (2010) ${ }^{1}$ und Rochkind (2013) $)^{2}$ beschriebenen Ansätzen werden wir im Folgenden die Architektur unseres Prototyps beschreiben, die angebotenen APIs (Application Programming Interfaces) und Metadaten und auch die Erfahrungen, welche die UB-Mitarbeiter und die regulären Bibliotheksbenutzer mit diesen Diensten gemacht haben.

\section{Technische Architektur}

Da es sich bei den Discovery-Diensten um Anwendungen handelt, die auf aktueller Suchmaschinentechnologie basieren, werden die Daten des Bochumer OPAC in einem täglich ablaufenden ETL-Prozess in eine Apache Solr-Instanz überführt. Während Summon ebenfalls Solr als Plattform nutzt, gibt es von EBSCO keine konkreten Informationen über die eingesetzte Technik. Da bei ProQuest an dieser

1 Jansen, Heiko; Kemner-Heek, Kirstin; Schweitzer, Roswitha: Konkurrenzanalyse ausgewählter kommerzieller Suchindizes. http://www.hbz-nrw.de/dokumentencenter/veroeffentlichungen/ suchindizes.pdf [Zugriff, auch bei allen folgenden Links: 15.07.2014].

2 Rochkind, Jonathan: A Comparison of Article Search APIs via Blinded Experiment and Developer Review. http://journal.code4lib.org/articles/7738. 
Stelle Open-Source-Software zum Einsatz kommt, kann man als Kunde gut den zu erwartenden Funktionsumfang der Plattform einschätzen, da sie sowohl im Web als auch in der Literatur ausführlich dokumentiert ist.

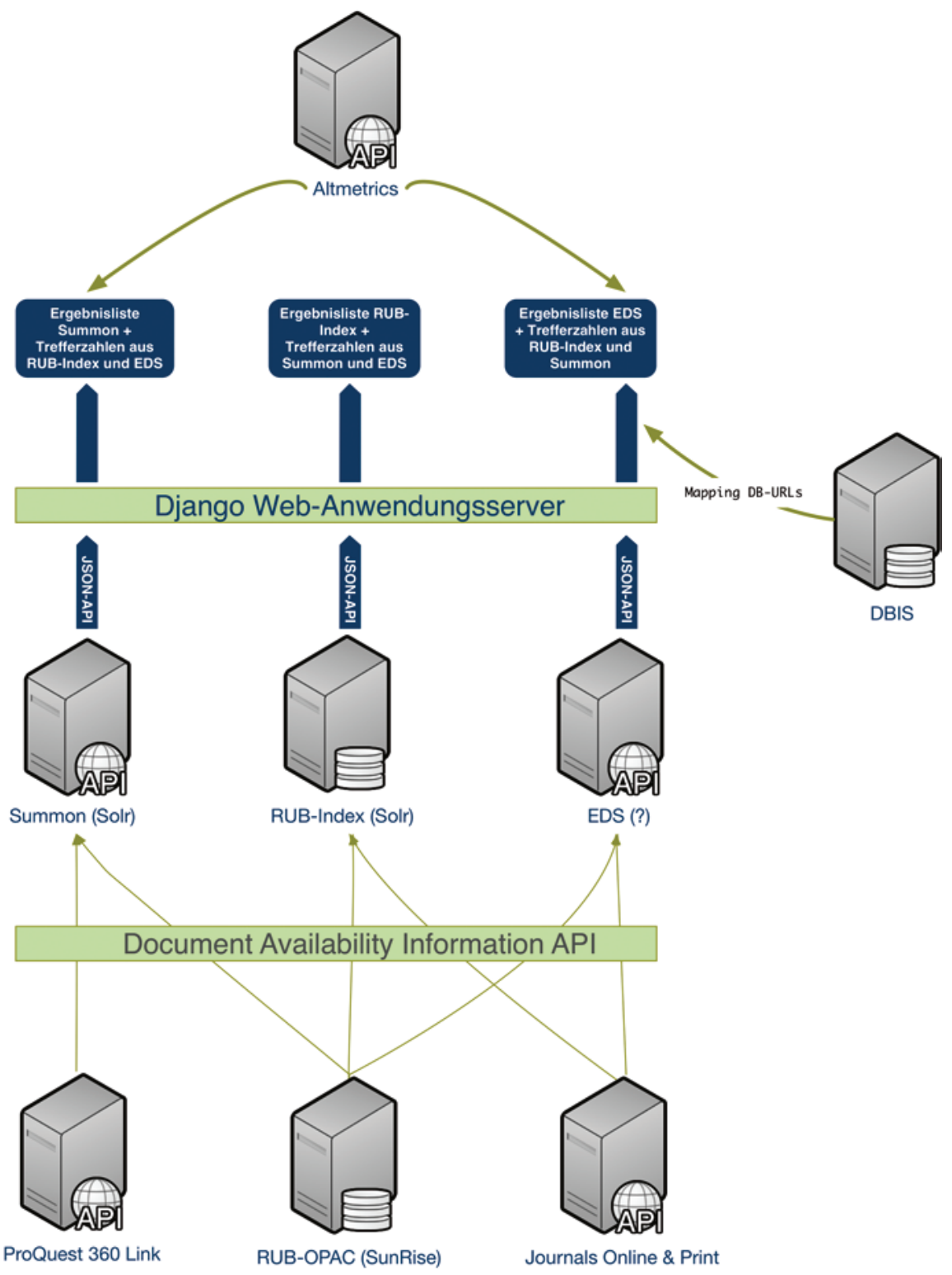

Abb.1: Diagramm der technischen Architektur. 
Sowohl Summon als auch EDS bieten ihren Kunden die Wahl zwischen der Nutzung als SaaS (Software as a Service), bei der die Webanwendung bei den Firmen gehostet wird und nur in geringem Umfang an die lokalen Bedürfnisse (sowohl in Bezug auf das Layout als auch die Funktionalitäten) angepasst werden kann, und der Einbindung des Dienstes über eine API. Da auch die Testumgebung möglichst neutral gestaltet werden sollte, wurde die letztere Möglichkeit gewählt. Kernstück der Architektur war ein Django Webapplikationsserver, der sowohl die Discovery-Dienste als auch den lokalen Index anspricht. Für das Oberflächenlayout kam das CSS-Framework Bootstrap ${ }^{3}$ in einer nur rudimentär an das Corporate Design der RUB angepassten Form zum Einsatz. Darüber hinaus stellte ein weiterer Django-Server eine Implementierung der Document Availability Information API (DAIA) ${ }^{4}$, die sowohl Verfügbarkeitsinformationen aus dem Lokalsystem als auch aus dem Journals Online \& Print (JOP)-Dienst der DNB als auch dem 360 Link-Resolver von ProQuest hinter dieser plattformneutralen Programmierschnittstelle kapselte. War es nicht möglich, über diese Dienste die Verfügbarkeit eines Titels zu ermitteln, wurde anhand der in den APIs der DiscoveryDienste enthaltenen OpenURL-Informationen ein Link zur Fernleihe der DigiBib erzeugt. Damit sollte den Nutzern die Möglichkeit gegeben werden, sofort anhand der Verfügbarkeit über die Relevanz eines Treffers zu entscheiden. Eine weitere Entscheidungshilfe über die Relevanz des Treffers wurde über die Integration des Dienstes Altmetric ${ }^{5} \mathrm{zu}$ geben versucht, der Metriken über die Verwendung eines Artikels im Web aggregiert.

Ein weiteres Desiderat dieses Tests sah vor, dass die Ergebnisse aus den Suchen in den Discovery-Diensten untereinander, aber auch zu unserem OPAC vergleichbar sein sollten. Daher haben wir die Trefferlisten so modelliert, dass eine Anfrage in eines der drei Systeme die Trefferanzahl der jeweils anderen beiden sowie einen Link mit der Suchanfrage in den jeweiligen Dienst lieferte. Dabei haben wir uns auf die reine Zeichenkette der Benutzeranfrage beschränkt, da die Navigatoren der drei Systeme nicht aufeinander abbildbar waren. Während unser lokaler Index über Navigatoren für Personen und Körperschaften verfügte, die sich aus der GND speisen, bot keiner der beiden Discovery-Dienste diese Kategorien an, zumal in keinen von ihnen Normdaten integriert sind. Ein hilfreicher Navigator, den beide Dienste bereitstellten, war die Möglichkeit, die Treffermenge auf peer reviewed-Artikel einschränken zu können. Da Summon diese

3 Twitter: Bootstrap. http://getbootstrap.com/2.3.2/.

4 Voss, Jacob; Reh, Uwe: Document Availability Information API (DAIA). http://gbv.github.io/ daiaspec/daia.html.

5 Altmetric: Altmetric API documentation. https://api.altmetric.com/. 


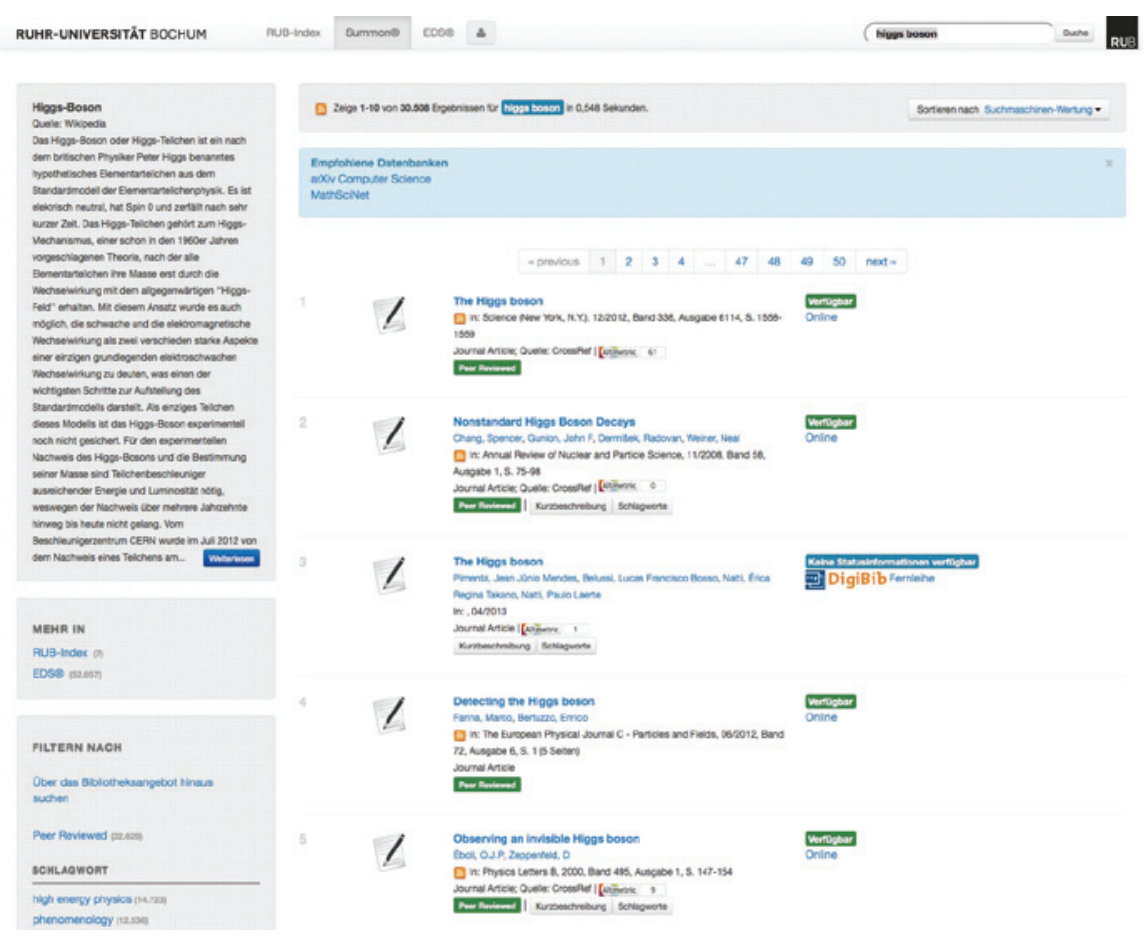

Abb. 2: Ergebnisliste in Summon.

Kategorie auch in den einzelnen Datensätzen zur Verfügung stellte, konnte diese Information auch prominent in den Treffern der Ergebnisliste dargestellt werden. Summon bietet darüber hinaus die Möglichkeit, enzyklopädische Informationen - aus der Encyclopedia Britannica oder der Wikipedia - in die Trefferliste zu integrieren. EDS zog diese Funktionalität zum Ende unserer Testperiode mit den Research Starters nach, jedoch fehlte uns die Zeit, diese zu implementieren und zu testen. Da in EDS die Zugehörigkeit eines Datensatzes zu der Datenbank, aus der er stammt, abzulesen war, haben wir anhand des Datenbanktitels ein semiautomatisches Mapping der Titel auf die URLs aus DBIS erstellt, sodass Nutzer sofort in der jeweiligen Datenbank weiterrecherchieren konnten. Da die Discovery-Dienste für sich in Anspruch nehmen, mit einer Google-artigen Ein-SchlitzSuche sehr gute Ergebnisse zu erzielen, haben wir auf die Implementierung einer erweiterten Suche verzichtet. 


\section{Metadaten und APIs}

ProQuest bezieht seine bibliographischen Daten direkt von den Verlagen, in EDS hingegen stammen diese von Datenbankanbietern, woraus sich einerseits Unterschiede im Umgang mit den Metadaten ergeben, anderseits schlägt sich dieser Umstand in zwei unterschiedlichen lizenzrechtlichen Modellen nieder. ProQuest setzt ein sogenanntes Match-\&-Merge-Verfahren ein, bei dem Dubletten von Titeldatensätzen verschiedener Anbieter zu einem einzigen Datensatz verschmolzen und dadurch angereichert werden. In EDS bleiben die Dubletten im Index nebeneinander bestehen, den Nutzern wird der umfangreichste Datensatz geliefert. ProQuest setzt also darauf, möglichst viele Informationen zu einem Titel in einem Datensatz zu vereinen, EBSCO indes legt Wert darauf, dass die Integrität der Datensätze erhalten bleibt und diese so angezeigt werden wie in den Datenbanken, aus denen sie stammen. Bei diesem Vorgehen besteht zwar die Möglichkeit, vom Titel direkt in die entsprechende Datenbank zu wechseln, andererseits entgehen den Nutzern Informationen (z. B. Schlagwörter), die sich nicht beim umfangreichsten Titel befinden und somit nicht angezeigt werden. ProQuest ermöglicht seinen Nutzern im Übrigen durch passend zur Suchanfrage generierte Datenbankvorschläge den Übergang in Datenbankangebote. Hierbei traten allerdings Probleme bei der Recherche mit deutschsprachigen Suchbegriffen auf, was darin begründet liegt, dass die Datenbankvorschläge aus Voreinstellungen und Crowdsourcing generiert werden. So wurden beispielsweise bei der Suche nach „epilepsy“ zwei passende Datenbanken angeboten, während die Suche nach „Epilepsie“ keine Datenbankvorschläge erzeugte.

Aus dem unterschiedlichen Umgang der beiden Discovery-Anbieter mit den Metadaten ergeben sich auch lizenzrechtliche Konsequenzen für die Nutzer dieser Angebote: Während die Metadaten in den Ergebnislisten in Summon vollständig angezeigt werden, sodass die Nutzer sofort und auf einen Blick ihr Suchergebnis bewerten können, werden die Trefferlisten in EDS unter Umständen nur authentifizierten Nutzern vollständig angezeigt. Technisch wäre die Anzeige der Metadaten in EDS problemlos möglich, allein aufgrund vertraglicher Vereinbarungen sehen Nutzer, die nicht durch Authentifizierung oder aufgrund ihrer IPAdresse autorisiert sind, lediglich Stellvertreter anstelle der Titeldaten, was den Benutzererwartungen an Suchanwendungen im Web fundamental widerspricht. 


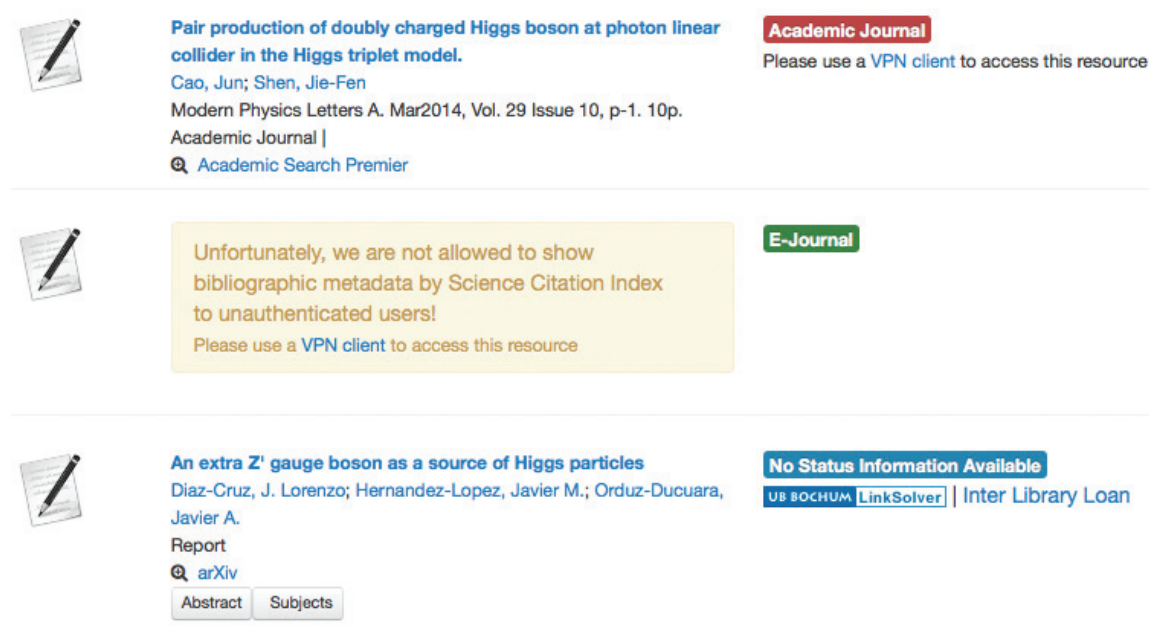

Abb. 3: Platzhalter für nicht frei anzeigbare Metadaten in EDS.

Während sowohl Summon als auch EDS eine REST-basierte API anbieten, deren Daten im XML- bzw. im JSON-Format ausgegeben werden, besteht der grundlegende Unterschied zwischen diesen beiden Angeboten darin, dass die SummonWebanwendung vollständig auf der API beruht, während die EDS-Oberfläche eine von den Programmierschnittstellen losgelöste Entwicklung darstellt. Während ProQuest mit der Summon-API „dogfooding“ betreibt, also den Einsatz des eigenen Produkts als Basis für andere eigene Produkte, um zu zeigen, dass man von dessen Qualität und Leistungsvermögen überzeugt ist, entstand die EDS-API eher im Nachgang zur Webanwendung.

Obschon die Dokumentation der Summon-API vollständig im Web für jedermann einsehbar ist und dort sehr übersichtlich die Komponenten und Parameter beschreibt, ist die EDS-API-Dokumentation nur Kunden zugänglich. Sie gliedert sich in zwei Dokumente, eine nutzerorientierte Übersicht und eine Referenz, die zwar detailliert über die Parameter Auskunft gibt, nicht jedoch über die Benennungen der im Index vorhandenen Felder. Das führte dazu, dass wir erst im Laufe unserer Implementierung durch Anschauung der konkreten Daten herausfinden konnten, in welchen Kategorien welche Werte zu erwarten waren. Wenig hilfreich war in diesem Zusammenhang, dass ein und dieselbe Kategorie mehrere Bezeichnungen besitzt. Ein Pluspunkt gegenüber der Summon-Dokumentation 
stellt hingegen die API-Console ${ }^{6}$ dar, in der man unterschiedlichste Anfragen ausprobieren kann.

Problematisch ist an der EDS-API allerdings, dass es vier Detailgrade bezüglich des Umfangs der Feldstruktur eines Datensatzes gibt: „Title“ (nur der Titel), „Brief“ (Titel, Quelle und Schlagwörter) und „Detailed“ (Brief plus Abstract). Während diese drei Ebenen der „Search“-Funktion der Ergebnisliste vorbehalten sind, gibt es den vollumfänglichen Datensatz nur mit der „Retrieve“-Funktion, die für die Vollanzeige genutzt wird. Eine solche Unterscheidung gibt es in Summon nicht: Dort ist schon in der Ergebnisliste jeder Treffer im vollen Umfang enthalten, weshalb sich dadurch Oberflächen implementieren lassen, die ohne eine eigene Vollanzeige auskommen. Das Fehlen einer einheitlichen Feldstruktur macht sich v. a. in der Vollanzeige von EDS negativ bemerkbar: Würde man hier der Empfehlung von EBSCO folgen, einfach alle Elemente in der Reihenfolge auszugeben, wie sie in der Antwort des Retrieve-Requests geliefert werden, könnte man keine mehrsprachigen Oberflächen, keine Mashups mit anderen Diensten und auch keine Verfügbarkeitsinformationen in der Vollanzeige realisieren. Um eine flexiblere Lösung umsetzen zu können, haben wir selbst ein Mapping aus den Daten abgeleitet und in einem iterativen Prozess ergänzt und verbessert.

Beide APIs liefern als Antwortformate sowohl XML als auch JSON aus. Da sich Letzteres einerseits in unserer Anwendung schneller verarbeiten ließ, andererseits von vielen als das Datenformat für das Web angesehen wird, haben wir uns zur Verwendung dieses Formats entschieden. Beiden Diensten ist gemein, dass sie für die Anzeige von Abstracts HTML in das Ausgabeformat einbetten, was man dann (solange es wohlgeformt ist) zur Anzeige bringen oder aus dem Output entfernen kann. Im Falle von EDS ist es allerdings dem Umstand geschuldet, dass dort das JSON aus dem XML-Format abgeleitet wird, dass HTML-Entitäten so codiert sind, dass sie von gängigen Web-Frameworks falsch interpretiert werden und zur fehlerhaften Darstellung in der Oberfläche führen. Darüber hinaus enthält das JSON in EDS einiger Schlagwortkategorien XML, das nur für das XMLAusgabeformat Sinn macht. Somit wird unnötig zusätzlicher Aufwand für die Bereinigung der Daten innerhalb der Webanwendung generiert. Insgesamt ist festzuhalten, dass der Einsatz der EDS-API im Vergleich zur Summon-API in ca. $10 \%$ umfangreicherem Code resultiert, den man als Benutzer der API schreiben und pflegen muss.

6 EBSCO: EDS API Console. https://eds-api.ebscohost.com/Console/. 


\section{Nutzererfahrungen}

Mit der Einbindung der beiden Discovery-Dienste EDS und Summon neben dem suchmaschinenbasierten OPAC unter einer gemeinsamen Benutzeroberfläche sollten den Nutzern nicht nur möglichst viele Datenquellen unter einer einheitlichen Oberfläche angeboten werden, sondern auch der Vergleich der beiden Discovery-Systeme sollte erleichtert und transparenter gestaltet werden.

Auf der Startseite der UB hatten die Nutzer zwei Möglichkeiten zum Einstieg in „suchen“: zum einen über einen Suchschlitz, über den der RUB-Index durchsucht wurde. In der Trefferliste konnten die Nutzer über die Reiter im Header zwischen den drei Angeboten wechseln. Zum anderen konnte „suchen“ über einen Link auf der Startseite der UB geöffnet werden. Umrahmt wurden Suchschlitz und Link von einem erklärenden Text, in dem gleichzeitig um Nutzerfeedback gebeten wurde. Hierbei kam der Online-Dienst Disqus ${ }^{7}$ zum Einsatz, der Betreibern und Nutzern von Websites eine zentralisierte Diskussions-Plattform bietet. $\mathrm{Zu}$ einem späteren Zeitpunkt wurden gezielt Studierende angeschrieben und um Feedback gebeten, wobei es insbesondere um die Entscheidung zugunsten eines der beiden Discovery-Services ging.

Leider gab es von Studierenden kaum Rückmeldungen zu „suchen“. Das meiste Feedback kam aus der UB selbst, und zwar aus den Fachreferaten, vom Zeitschriftenteam, dem Schulungsteam sowie den Auszubildenden. Geisteswissenschaftliche Themen bildeten den größten Teil des Feedbacks, wenngleich es vereinzelt Rückmeldungen zu naturwissenschaftlichen Recherchen gab. Im Vordergrund standen dabei weniger Usability-Aspekte als vielmehr die Qualität der angebotenen Inhalte. Alles in allem konnte das abgegebene Feedback nicht als repräsentativ angesehen und dementsprechend nicht statistisch ausgewertet werden, jedoch waren gewisse Tendenzen zu beobachten, die im Folgenden kurz aufgezeigt werden sollen.

Die Studierenden und die Auszubildenden lobten die einfache Bedienung und die Übersichtlichkeit der Anwendungsoberfläche. Das Angebot wurde als optisch ansprechender als der klassische OPAC empfunden. Als besonders positiv hervorgehoben wurde von dieser Zielgruppe der Umstand, dass in den Discovery-Angeboten mit einer Suchanfrage gleichzeitig verschiedene Medienformen gefunden werden, die zuvor mühsam separat - Bücher in Katalogen, Aufsätze in Datenbanken - ermittelt werden und auf ihre Verfügbarkeit überprüft werden mussten. In „suchen“ ist es nicht nur möglich, zwischen den Ergebnislisten der beiden Discovery-Dienste sowie den OPAC-Daten hin- und herzuwech-

7 Disqus: Disqus - The Web's Community of Communities. https://disqus.com/. 
seln, sondern auch die Verfügbarkeit wird direkt in der Kurztrefferliste angezeigt. Die Nutzer müssen also nicht die Vollanzeige jedes einzelnen Titels überprüfen, sondern sehen sofort, welche Titel für sie verfügbar sind. Studierende und Auszubildende sprachen sich mehrheitlich dafür aus, dass die UB einen DiscoveryDienst anbieten sollte, lediglich aus dem Fach Mathematik kam die Rückmeldung, dass dort kein Bedarf an Discovery bestehe - eine Position, die in der UB von den meisten Fachreferenten der naturwissenschaftlichen Fächer geteilt wurde. In den geisteswissenschaftlichen Fächern wurden die Vorteile eines Angebotes, das verteilte Quellen zusammenführt, gesehen, allerdings wurde die intuitiv benutzbare Oberfläche mit Skepsis betrachtet und der Wunsch nach einer Recherchemaske für eine erweiterte Suche geäußert. Vor allem aber wurden Bedenken bezüglich der inhaltlichen Qualität der Discovery-Angebote bzw. des Relevanzrankings formuliert.

Beide Discovery-Dienste unter derselben Oberfläche wie den OPAC anzubieten, war insofern sinnvoll, als dass deckungsgleiche Strukturen auch als solche identifiziert werden konnten und Unterschiede zwischen den Produkten deutlicher hervortraten. Am auffallendsten waren zunächst aus den APIs resultierende Unterschiede, u. a. der Umgang mit Dubletten ${ }^{8}$, die - in EDS aufgrund fehlender Dokumentation von Feldern - problematische Darstellung von Abstracts und eine unterschiedliche Facettierung der Ergebnismenge. In Summon haben die Nutzer die Möglichkeit, nach peer reviewed, Schlagwort, Fachbereich, Publikationstyp, Sprache und Jahr zu filtern. In EDS wird die Treffermenge teilweise nach anderen Kriterien facettiert: Nutzer können hier nach peer reviewed, Schlagwort, Veröffentlichung (d.h. dem übergeordneten Titel), Publikationstyp, Sprache, Verlag und Datenquelle filtern; ein Navigator für das Erscheinungsjahr existiert bedauerlicherweise nicht bzw. dieser ist nur in der originären EDS-Oberfläche vorhanden - über die EDS-API werden diese Informationen nicht geliefert. Das Fehlen dieses Navigators wurde von den Nutzern kritisiert, die Filter Veröffentlichung und Verlag wurden als überflüssig charakterisiert. Der Link zu den Datenbanken fand hingegen Anklang, ebenso wie der Schlagwort-Navigator.

Hinsichtlich der Zusatzfeatures, die in die Discovery-Dienste eingebunden werden können, sticht vor allem die Möglichkeit hervor, in Summon Kontextinformationen in Form von gemeinfreien, offen lizenzierten Inhalten (Wikipedia) zur Recherche anzubieten. Die Nutzer (in erster Linie Studierende und Auszubildende) äußerten sich positiv über dieses Feature.

8 In beiden Discovery-Systemen traten Dubletten auf; kritisiert wurde in erster Linie deren Häufung in EDS. 


\section{Fazit}

Durch die Integration der unterschiedlichen Angebote in einer Webanwendung mit einer gemeinsamen Suchoberfläche war es möglich, die getesteten Dienste unabhängig von ihrem äußeren Design zu testen und strukturelle Unterschiede sichtbar zu machen, um die dabei gemachten Beobachtungen für eine Entscheidungsfindung zu nutzen. Eine technische Lösung wie der hier vorgestellte Prototyp unterstützt kurzfristig den Vergleich und die Evaluierung unterschiedlicher Dienste, langfristig ist jedoch eine Lösung wünschenswert, in der die Nutzer nicht via Reiter zwischen den Datenquellen hin- und herwechseln müssen, sondern in der möglichst alle Daten in einem einzigen Index integriert und somit auch gemeinsam durchsuch- und facettierbar sind. Der nächste Schritt wäre also nach der Entscheidung für eines der beiden Discovery-Systeme - die Integration der OPAC-Daten in den Index des gewählten Discovery-Dienstes. Eine solche Integration ist bei beiden getesteten Discovery-Systemen prinzipiell möglich, würde sich jedoch aufgrund der Metadatenstrukturen und der API-Architektur mit Summon unkomplizierter gestalten als in EDS. Unabhängig von der Entscheidung hinsichtlich der Discovery-Services wird „suchen“ auch in Zukunft weiterentwickelt werden, um den Nutzern einerseits die OPAC-Daten auf der Basis von Suchmaschinentechnologie zugänglich zu machen und ihnen durch Mashups mit anderen Diensten Funktionalitäten anzubieten, die über den traditionellen OPAC hinausgehen. Andererseits müssen grundlegende Benutzerfunktionalitäten wie Vormerkung oder Verlängerung implementiert werden, sodass ein solcher Dienst den lokalen OPAC ablösen könnte. Es stellt sich zudem die Frage, ob weitere Inhalte in den RUB-Index eingebunden werden sollen bzw. welche Inhalte dabei relevant wären und den Nutzern einen Mehrwert böten.

Führt man den Gedanken der Integration möglichst vieler Datenquellen in ein einziges Angebot weiter, kann ein Discovery-Dienst allerdings keine endgültige Lösung sein, da es sich bei Discovery-Diensten ebenfalls um nichts anderes handelt als die Datensilos ${ }^{9}$, mit denen Bibliotheken seit Jahrzehnten operieren wenngleich diese herkömmliche Kataloge und Datenbanken in ihren Dimensionen um ein Vielfaches übertreffen. Um den jetzigen Zustand der verteilten Suche in unterschiedlichen Informationsangeboten konsequent zu überwinden, werden Bibliotheken Webstandards, Linked (Open) Data und Suchmaschinentechnologie nicht nur in ihre eigenen Anwendungen einbringen müssen, sondern sich mit

9 Vgl. zu einer daten- statt containerbasierten Haltung auch Koster, Lukas: Old silos, new silos, no silos. SWIB 2012, Köln, 26.-28.11.2012. http://de.slideshare.net/lukask/old-silos-new-silosno-silos. 
ihren Metadaten und Informationsangeboten wie Katalogen und Datenbanken ins Web begeben müssen. Auch wenn mit Discovery-Systemen eine Recherche im Web simuliert wird - letztlich wird erst im Web selbst eine wirklich integrierte Suche über einen einzigen Index möglich sein.

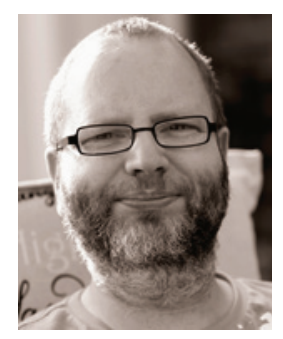

\section{André Hagenbruch}

Universitätsbibliothek Bochum

44780 Bochum

E-Mail: andre.hagenbruch@rub.de

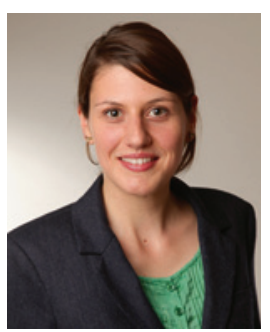

\section{Miriam Heise}

Universitätsbibliothek Bochum

44780 Bochum

E-Mail: miriam.heise@rub.de 